\title{
Expression levels of UL16 binding protein 1 and natural killer group 2 member $D$ affect overall survival in patients with gastric cancer following gastrectomy
}

\author{
RYOJI KAMEI $^{1}$, KIYOSHI YOSHIMURA ${ }^{1-3}$, SHIGEFUMI YOSHINO ${ }^{1}$, MOEKO INOUE $^{2,3}$, TETSUHIKO ASAO $^{2,3}$, \\ MASANORI FUSE ${ }^{2,3}$, SATOSHI WADA ${ }^{4}$, ATSUO KURAMASU ${ }^{5}$, TOMOKO FURUYA-KONDO ${ }^{6}$, ATSUNORI OGA ${ }^{6}$, \\ NORIO IIZUKA $^{7}$, NOBUAKI SUZUKI ${ }^{1}$, NORIKO MAEDA ${ }^{1}$, YUSAKU WATANABE ${ }^{1}$, SATOSHI MATSUKUMA $^{1}$, \\ MICHIHISA IIDA $^{1}$, SHIGERU TAKEDA ${ }^{1}$, TOMIO UENO ${ }^{1}$, NOBORU YAMAMOTO ${ }^{2}$, TAKEO FUKAGAWA ${ }^{8}$, \\ HITOSHI KATAI $^{8}$, HIROKI SASAKI ${ }^{9}$, SHOICHI HAZAMA ${ }^{1}$, MASAAKI OKA ${ }^{1}$ and HIROAKI NAGANO ${ }^{1}$ \\ ${ }^{1}$ Department of Gastroenterological, Breast and Endocrine Surgery, Yamaguchi University School of Medicine, Ube, \\ Yamaguchi 755-8505; ${ }^{2}$ Experimental Therapeutics, National Cancer Center Hospital; ${ }^{3}$ Division of Cancer Immunotherapy, \\ Exploratory Oncology Research and Clinical Trial Center, National Cancer Center, Tsukiji, Chuo-ku, Tokyo 104-0045; \\ ${ }^{4}$ Division of Cancer Immunotherapy, Kanagawa Cancer Center, Yokohama, Kanagawa 241-8515; Departments of \\ ${ }^{5}$ Molecular Pharmacology and ${ }^{6}$ Molecular Pathology, Yamaguchi University Graduate School of Medicine, Ube, \\ Yamaguchi 755-8505; ${ }^{7}$ Department of KAMPO Medicine, Graduate School of Biomedical and Health Sciences, \\ Hiroshima University, Hiroshima, Hiroshima $734-0037 ;{ }^{8}$ Gastric Surgery Division, National Cancer Center Hospital \\ and ${ }^{9}$ Fundamental Innovative Oncology Core, Biomarker and Therapeutic Target Research Core, \\ National Cancer Center Institute, Chuo-ku, Tokyo 104-0045, Japan
}

Received August 3, 2016; Accepted June 9, 2017

DOI: 10.3892/ol.2017.7354

\begin{abstract}
UL16 binding protein 1 (ULBP1) expressed on the tumor cell surface binds to the natural killer group 2 member $\mathrm{D}(\mathrm{NKG} 2 \mathrm{D})$ receptor presenting on natural killer $(\mathrm{NK})$, cluster of differentiation (CD) $8^{+} \mathrm{T}$, and $\gamma \delta \mathrm{T}$ cells. However, the roles of ULBP1 and NKG2D expression and associated immune responses in gastric cancer are unclear. The present study investigated the associations between ULBP1 and NKG2D expression and clinical outcomes in patients with gastric cancer. The levels of ULBP1 and NKG2D expression were examined in human gastric cancer cell lines and gastric cancer tissues from 98 patients who underwent surgery from 2004 to 2008 . MKN-74 cells expressed ULBP1 with ULBP2, -5 , or -6 . NKG2D was expressed at a higher level following
\end{abstract}

Correspondence to: Dr Kiyoshi Yoshimura, Division of Cancer Immunotherapy, Exploratory Oncology Research and Clinical Trial Center, National Cancer Center, 5-1-1 Tsukiji, Chuo-ku, Tokyo 104-0045, Japan

E-mail: kiyoshim@ncc.go.jp

Abbreviations: ULBP1, UL16 binding protein 1; MICA, MHC class I chain-related proteins A; NKG2D, natural killer group 2 member D

Key words: NKG2D, NKG2D ligand, gastric cancer, ULBP1, biomarker activation of $\mathrm{T}$ cells and $\mathrm{NK}$ cells. Among the tissue sections positive for NKG2D expression, 6 patients were positive for CD8 and CD56. In all tissues, NKG2D-expressing cells were typically aCD $8^{+} \mathrm{T}$ cells. Patients with $\mathrm{NKG} 2 \mathrm{D}$ expression in tumors exhibited significantly longer overall survival (OS) compared with patients without NKG2D expression in tumors $(\mathrm{P}=0.0217)$. The longest $\mathrm{OS}$ was observed in patients positive for ULBP1 and NKG2D, whereas the shortest OS was observed in patients negative for ULBP1 and NKG2D. The interaction between ULBP1 and NKG2D may improve OS in patients with gastric cancer, and may have applications in immunotherapy for the induction of adaptive immunity in patients with cancer. Additionally, ULBP1 and NKG2D may be useful as prognostic biomarkers in gastric cancer.

\section{Introduction}

Gastric cancer is the fourth most common type of cancer, and the second leading cause of cancer-associated mortality worldwide (1). Therefore, novel therapies for the treatment of gastric cancer are urgently needed.

The expression of natural killer group 2 member D (NKG2D) on natural killer (NK) cells, $v \delta \mathrm{T}$ cells and $\mathrm{CD}^{+} \mathrm{T}$ cells was first identified in 1991 (2), and its function was subsequently reported in 1999 (3). A total of 6 UL16 binding proteins (ULBP1-6) and MHC class I chain-related proteins A and $\mathrm{B}(\mathrm{MICA} / \mathrm{B})$ are the two major types of NKG2D ligands (NKG2DLs) for human NKG2D. mRNA export factor, Mult1, and histocompatibility antigen 60 are the 3 major NKG2DLs for murine NKG2D (4). NKG2DLs are induced by cellular or 
genomic stress. When expressed on $\mathrm{CD} 8^{+} \mathrm{T}$ cells, NKG2D functions to receive co-stimulatory signals, thereby resulting in $\mathrm{CD}^{+}$T-cell activation (5). NKG2DL expression is induced upon stress and malignant transformation (6). In addition, NKG2D expression on mononuclear cells is significantly lower in patients with advanced gastric cancer compared with in patients with early gastric cancer. Additionally, NKG2D expression is significantly higher following surgery compared with prior to surgery in patients with gastric cancer (7). The upregulation of NKG2DLs in cancer cells subjected to stress results in lysis by NK cells (8).

The surface glycoprotein MICA is an NKG2DL broadly expressed in epithelial and hematopoietic tumors, but not in healthy tissue. Subsequent to shedding of MICA in tumors, soluble MICA functions in the immune escape of tumors. Importantly, the level of soluble MICA has been positively correlated with tumor node metastasis (TNM) stage in patients with breast cancer (9). Additionally, soluble MICA has been demonstrated to reduce the expression of NKG2D, impair NK-mediated immune surveillance, which leads to immune escape of breast tumors (9). Interleukin (IL)-12 may also upregulate the expression of NKG2D and increase cytotoxicity of NK cells (10). However, the potential applications of an additional key NKG2DL, ULBP1, as a biomarker or prognostic factor for patients with gastric cancer have not been determined, and the role of the ULBP1/NKG2D interaction in patients with gastric cancer is not yet clear.

The present study investigated whether ULBP1 and NKG2D expression levels were associated with the clinical outcomes of patients with gastric cancer.

\section{Materials and methods}

Gastric cancer cell lines and culture conditions. The human gastric cancer MKN-74 cell line, established at the National Cancer Center, was maintained in Dulbecco's modified Eagle's medium/F12 (Sigma-Aldrich; Merck KGaA, Darmstadt, Germany) supplemented with $10 \%$ heat-inactivated fetal bovine serum (FBS; Thermo Fisher Scientific, Inc., Waltham, $\mathrm{MA}, \mathrm{USA})$ at $37^{\circ} \mathrm{C}$ in an atmosphere containing $5 \% \mathrm{CO}_{2}$.

Patients. A total of 98 samples were obtained from patients who underwent surgery at the Department of Digestive Surgery and Surgical Oncology, Yamaguchi University Graduate School of Medicine (Yamaguchi, Japan) from January 2004 to December 2008. The patients were diagnosed with gastric cancer with the tumor invaded through the muscularis propria or deeper. No patients had received pre-operative therapy. Pathological staging was performed according to the Japanese Classification of Gastric Carcinoma (14th edition) based on the Japanese Gastric Cancer Association TNM staging system (11). The clinicopathological characteristics of the patients are summarized in Table I. The study protocol was approved by the Institutional Review Board for Human Use at Yamaguchi Hospital (Yamaguchi, Japan). Written informed consent for the present study was obtained from all patients prior to surgery.

Analysis of ULBP, MICA/MICB expression in cancer cell lines and NKG2D expression in NK/T cells by flow cytometric
Table I. Characteristics of the study population.

\begin{tabular}{lc}
\hline Variables & No. of cases \\
\hline $\begin{array}{l}\text { Number of patients } \\
\text { Age, years (range) }\end{array}$ & 98 \\
$\begin{array}{l}\text { Gender } \\
\text { Male/Female }\end{array}$ & $64 \pm 14(23-92)$ \\
Depth of tumor invasion & $65 / 33$ \\
MP/SS/SE/SI & \\
H. & $21 / 25 / 49 / 3$
\end{tabular}

$\mathrm{MP} / \mathrm{SS} / \mathrm{SE} / \mathrm{SI}$

$3 / 28 / 51 / 9 / 7$

Papillary adenocarcinoma/tubular

adenocarcinoma/poorly differentiated

adenocarcinoma/signet-ring cell

carcinoma/mucinous adenocarcinoma

Stroma

Medullary type/interstitial connective

tissue/scirrhous type

Pattern of tumor infiltration

$\mathrm{a} / \mathrm{b} / \mathrm{c}$

$4 / 34 / 60$

Lymphatic invasion

$0 / 1 / 2 / 3$

$7 / 25 / 37 / 29$

Venous invasion

$0 / 1 / 2 / 3$

$23 / 50 / 21 / 4$

Regional lymph nodes

$\mathrm{N} 0 / \mathrm{N} 1 / \mathrm{N} 2 / \mathrm{N} 3$

$27 / 20 / 13 / 38$

Stage

$\mathrm{I} / \mathrm{II} / \mathrm{III} / \mathrm{IV}$

$11 / 30 / 34 / 23$

MP, muscularis propria; SS, subserosa; SE, serosal exposure; SI, invasion.

analysis. Flow cytometric analysis was performed to confirm the expression of NKG2DLs on the MKN-74 gastric cancer cell and NKG2D on the surface of the NK and T cells. BD FACSFlow (BD Biosciences, San Jose, CA, USA) was used for buffer of flow cytometric analysis. ULBP1 against MKN-74 was stained using phycoerythrin (PE)-conjugated mouse anti-ULBP1 antibody (dilution, 1:10; cat no. FAB1380P; R\&D Systems, Inc., Minneapolis, MN, USA), and ULBP $2 / 5 / 6$ against MKN-74 was stained using allophycocyanin (APC) -conjugated mouse anti-ULBP2/5/6 (dilution, 1:10; cat no. FAB1298A; R\&D Systems, Inc.). CD3 against NK and $\mathrm{T}$ cells was stained using v450-conjugated mouse anti-CD3 (dilution, 1:20; cat no. 560351; BD Biosciences), CD8 against NK and T cells was stained using APC-Cy7-conjugated mouse anti-CD8 (dilution, 1:20; cat no. 557834; BD Biosciences), CD16 against NK and T cells was stained using v500-conjugated mouse anti-CD16 (dilution, 1:20; cat no. 561394; BD Biosciences), and CD56 against NK and T cells was stained using PE-CF594-conjugated mouse anti-CD56 (dilution, 1:20; cat no. 562289; BD Biosciences). All samples were maintained at $4^{\circ} \mathrm{C}$ for $30 \mathrm{~min}$. MKN-74, mononuclear cells, activated $\mathrm{T}$ cells and activated NK cells were stained to identify each cell surface markers. Following 3 washes with BD FACSFlow 
buffer, the cells were resuspended in phosphate-buffered saline (PBS) with $2 \% \mathrm{FBS}$ at a density of $2 \times 10^{5}$ cells $/ 100 \mu 1$. Appropriate isotype control antibodies were stained in the aforementioned manner.

Samples were analyzed with a BD LSRFortessa X-20 instrument (BD Biosciences), and data were analyzed with FlowJo software version x10.0.7r2 (Tree Star, Inc., Ashland OR, USA). The relative fluorescence intensity (RFI) was calculated as follows: RFI $=[(\mathrm{MFI})$ of molecule $)-(\mathrm{MFI}$ of corresponding isotype control)]/MFI of corresponding isotype control, where MFI is the mean fluorescence intensity.

NK and T cell activation. Mononuclear cells (LP_241; Cellular Technology, Ltd., Cleveland, OH, USA) were activated to generate NK cells by addition of $5 \mu \mathrm{l} / \mathrm{ml} /$ well of mixed CD2 and CD335 beads with $500 \mathrm{U} / \mathrm{ml} /$ well IL-2 (all from Miltenyi Biotec GmbH, Bergisch Gladbach, Germany) for 2 h. For T-cell activation, the mononuclear cells were stimulated with $2 \mu \mathrm{g} / \mathrm{ml} /$ well anti-CD3 agonistic antibodies (eBioscience; Thermo Fisher Scientific, Inc.) and $1 \mu \mathrm{g} / \mathrm{ml} /$ well anti-CD28 agonistic antibodies (BioLegend, San Diego, CA, USA) with $100 \mathrm{U} / \mathrm{ml} /$ well IL-2 (Miltenyi Biotec $\mathrm{GmbH}$ ) for $2 \mathrm{~h}$.

Immunohistochemistry (IHC). Paraffin-embedded tissue blocks were cut into $5 \mu \mathrm{m}$ sections, mounted on saline-coated slides, and subsequently dewaxed and rehydrated using xylene and graded alcohol washes. Antigen retrieval was performed by microwaving in Target Retrieval solution ( $\mathrm{pH}$ 9; Dako; Agilent Technologies, Inc., Santa Clara, CA, USA). Endogenous peroxidase was blocked at room temperature (RT) for 15 min with $0.3 \%$ hydrogen peroxide (Wako Pure Chemical Industries, Ltd., Osaka, Japan) in PBS containing $0.1 \%$ sodium azide. Subsequent to washing twice in PBS, the sections were blocked with Protein Block Serum-Free (Dako; Agilent Technologies, Inc.) at RT for $30 \mathrm{~min}$. The primary antibodies were then added. Anti-ULBP1 antibodies (cat no. HPA007547; 1:100; Sigma-Aldrich; Merck KGaA), anti-NKG2D antibodies (cat no. ab36136; 1:100; Abcam, Cambridge, UK), anti-CD56 antibodies (cat no. B159; 1:50), and anti-CD8 antibodies (cat no. SK1; 1:50) (both from BD Biosciences) were used for IHC according to the manufacturers' protocol. Briefly, following incubation with primary antibodies at $4^{\circ} \mathrm{C}$ for $15 \mathrm{~h}$, the slides were washed twice in PBS, incubated with horseradish peroxidase-conjugated rabbit/mouse antibodies with original concentration at RT for $30 \mathrm{~min}$ (K406311-2; EnVision Plus mouse/HRP system; Dako; Agilent Technologies, Inc.), incubated with Dako-Chromogen solution, and washed in de-ionized water. Following background staining with Mayer's hematoxylin, the sections were dehydrated through ascending alcohols to xylene and mounted. To confirm the specificity of antibodies, negative control slides were incubated with mouse IgG1 monoclonal antibodies (ab81032; 1:100; Abcam). IHC results were evaluated by imaging under low-power magnification (x100) in eight fields of view to identify regions containing positive immunoreactivity. Immunostaining was additionally evaluated at a high-power (x400) magnification (ECLIPSE E200 and Nikon 1 V2 Micro imaging system; Nikon, Tokyo, Japan). Microscopic analysis of ULBP1 and NKG2D expression was performed by three blinded independent observers.
Scoring of ULBP1 expression in cancer cells. Evaluation of ULBP1 staining in the cancer cells was performed semi-quantitatively, as described previously by Loos et al (12) Evaluation of ULBP1 staining was based on the area and intensity of the staining. Scoring system for area of staining is as follows: Score $1,<33 \%$ of cancer cells; score $2,33-66 \%$ of cancer cells; score $3,>66 \%$ of cancer cells. Scoring for staining intensity is as follows: Score 1, absent/weak staining; score 2, moderately intense staining and score 3 , strong staining. Each section was given a final grade derived from the combination of the area and intensity scores. The final score was calculated by adding scores of intensity and area. Sections with a final score of 3 were classified as exhibiting low ULBP1 expression (ULBP1 low), whereas sections with a final score of $>3$ were classified as exhibiting high ULBP1 expression (ULBP1 high).

Scoring of NKG2D expression on mononuclear cells around gastric tumors. Absolute numbers of NKG2D-positive cells in $1 \mu \mathrm{m}$ invasive tumor core biopsies were counted manually using an eyepiece reticule by three blinded independent individuals. The number of NKG2D-positive cells around the tumors was counted using a computerized image analysis system composed of an Olympus DP70 CCD camera on an Olympus AX70 light microscope (Olympus, Tokyo, Japan). Under magnification, $\mathrm{x} 400$, there were $\geq 8$ independent and intact computerized microscopic fields for the duplicates of each patient sample. A total of four independent microscopic fields (magnification, $\mathrm{x} 400$ ), representing the densest lymphocytic infiltrates, were selected for each patient sample to ensure representativeness and homogeneity. The scores for four fields were averaged to calculate the final number for one computerized microscopic field at magnification, $x 400\left(0.0768 \mathrm{~mm}^{2} /\right.$ field $)$. The evaluation of NKG2D-positive cells was performed by three independent blinded observers. Discrepancies in enumeration, within a range of 5\%, were re-evaluated and a consensus decision was made. The ratio of NKG2D-positive cells/tumor-infiltrating lymphocytes (TILs) was calculated for each specimen. The median value was selected as the cut-off for defining TIL subgroups (median, 0.093). High and low ratios of NKG2D were termed NKG2D high and NKG2D low, respectively.

Statistical analysis. Differences were analyzed using Fisher's exact tests. Actuarial OS rates and recurrence-free survival rates were calculated by the Kaplan-Meier method and analyzed by the log-rank test. Univariate analyses were performed using Fisher's exact tests. Multivariate analyses were performed to determine factors affecting overall survival (OS) based on the Cox proportional hazards regression model. A secondary analysis was performed to assess the associations among the expression of NKG2DLs/NKG2D and clinicopathological characteristics by multivariate analysis based on the proportional hazards regression model. The activation of T cells and NK cells was analyzed by Mann-Whitney tests. For comparisons of individual variables, paired-sample t-tests were performed. Two-tailed $\mathrm{P}<0.05$ was considered to indicate a statistically significant difference. All statistical analyses were performed with EZR version 1.35 (Saitama Medical Center, Jichi Medical University), a graphical user interface for R (The R Foundation for Statistical Computing, v.2.13.0), and more specifically a modified version of $\mathrm{R}$ commander 


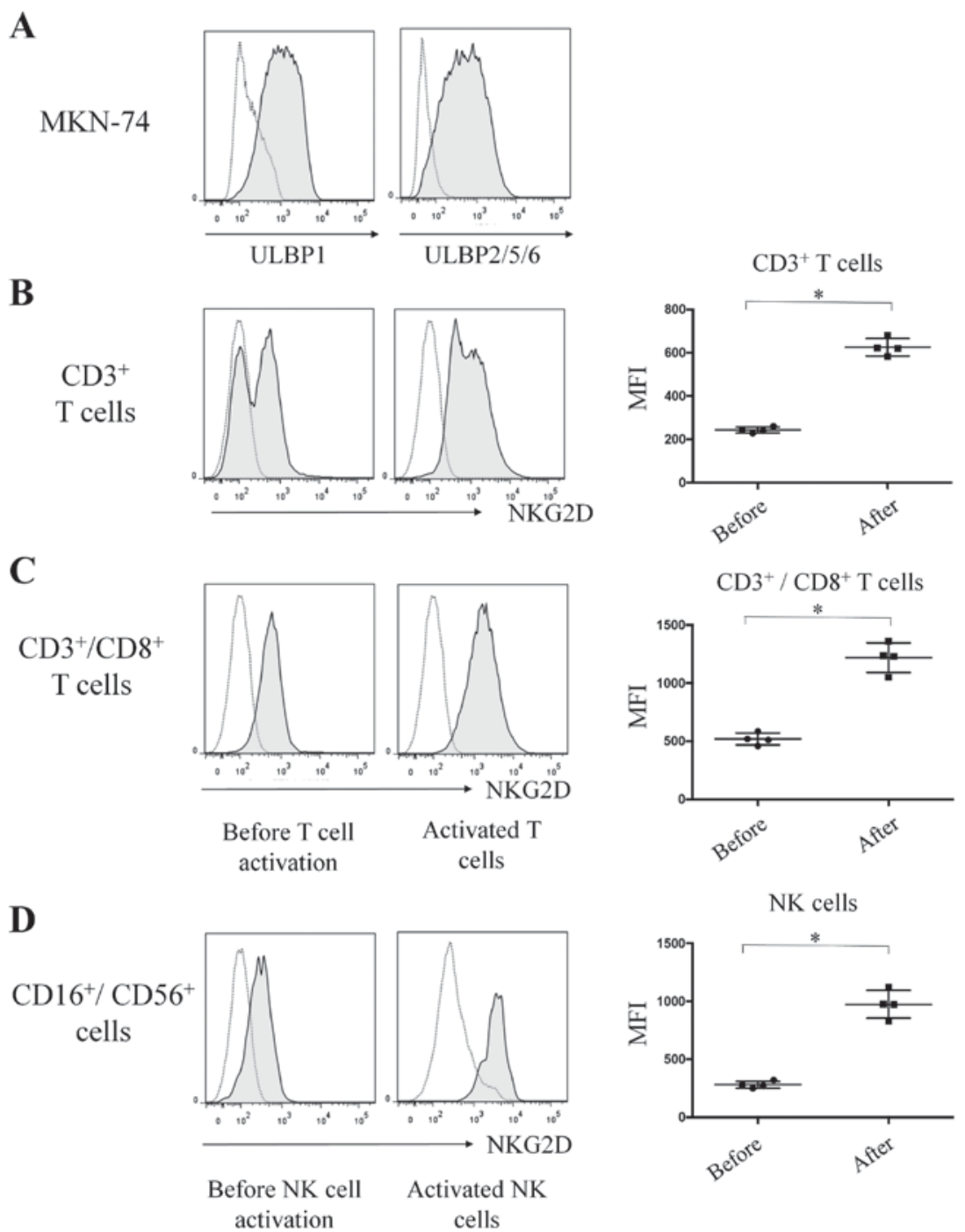

Figure 1. (A) ULBP1 and ULBP2/5/6 expression on the surface of MKN-74 cells. (B) NKG2D expression on CD3 ${ }^{+}$prior and subsequent to T-cell activation $(\mathrm{n}=4)$. (C) NKG2D expression on $\mathrm{CD}^{+} / 8^{+}$cells prior and subsequent to T-cell activation $(\mathrm{n}=4)$. (D) NKG2D expression on $\mathrm{CD} 16^{+} / \mathrm{CD}^{2} 6^{+}$cells prior and subsequent to NK cell activation $(n=4)$. "P<0.05. ULBP, UL16 binding protein; $C D$, cluster of differentiation; NKG2D, natural killer group 2 member $D$; MFI, mean fluorescence intensity; NK, natural killer.

(v.1.6-3) designed to add statistical functions frequently used in biostatistics.

\section{Results}

Expression of NKG2DLs in cancer cell lines. In the flow cytometric analysis, cell surface expression of ULBP1 and ULBP2/5/6 in MKN-74 cells was constitutively high (Fig. 1A). By contrast, cell surface expression of MICA and MICB was not detected in MKN-74 cells (data not shown).

Expression of NKG2D in mononuclear cells. The cell surface expression of NKG2D on $\mathrm{CD}^{+} \mathrm{T}$ cells was significantly increased following T-cell activation (MFI, 621) compared with the expression (MFI, 243) prior to T-cell activation $[\mathrm{P}=0.0286, \mathrm{n}=4$, standard deviation $(\mathrm{SD})=378$; Fig. 1B $]$. Similarly, cell surface expression of NKG2D on $\mathrm{CD} 3^{+} \mathrm{CD} 8^{+}$ $\mathrm{T}$ cells was significantly higher following T-cell activation (MFI, 1234) compared with expression (MFI, 515) prior to T-cell activation $(\mathrm{P}=0.0286, \mathrm{n}=4, \mathrm{SD}=719$; Fig. 1C). Cell surface expression of NKG2D on $\mathrm{CD} 16^{+} \mathrm{CD} 56^{+} \mathrm{NK}$ cells was significantly higher following NK cell activation (MFI, 974) compared with expression (MFI, 276) prior to NK cell activation $(\mathrm{P}=0.0283, \mathrm{n}=4, \mathrm{SD}=698$; Fig. 1D).

IHC staining of ULBPI. ULBP1 expression was observed in the cell membrane and cytoplasm. Fig. 2A and B show representative images of ULBP1-high staining in gastric cancer (magnification, x100 and x400, respectively). Fig. 2C and D show isotype control staining in gastric cancer (magnification, $\mathrm{x} 100$ and $\mathrm{x} 400$, respectively). Of the 98 samples examined in the analysis, 70 were positive for ULBP1 expression.

IHC staining of $N K G 2 D$. NKG2D was expressed on the membrane of mononuclear cells around the gastric tumors. Fig. 2E and $\mathrm{F}$ reveal high NKG2D expression in TILs with (magnification, $\mathrm{x} 100$ and $\mathrm{x} 400$, respectively). Fig. 2G and $\mathrm{H}$ demonstrate isotype control staining in TILs 
A

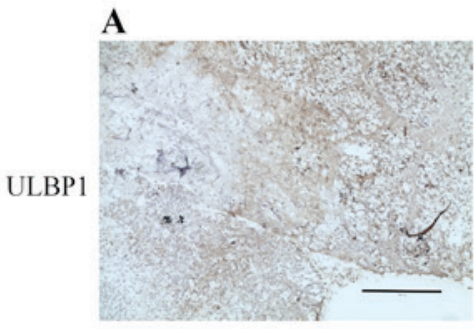

C

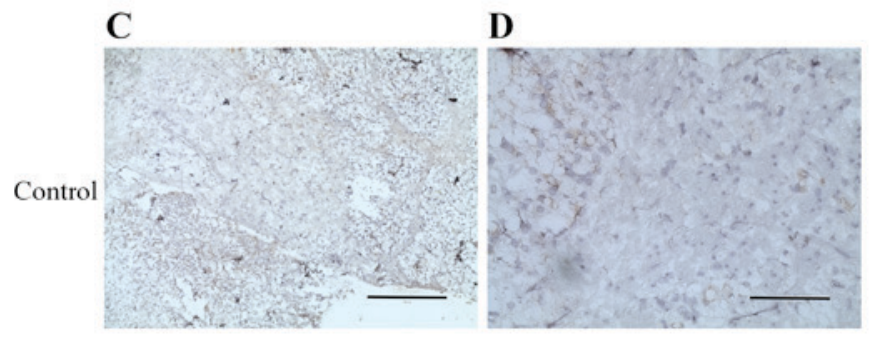

I

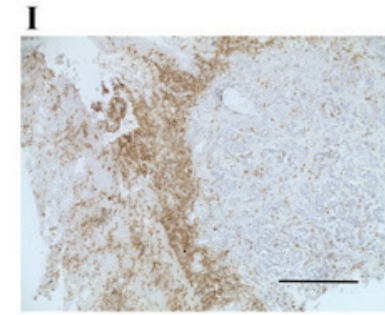

K

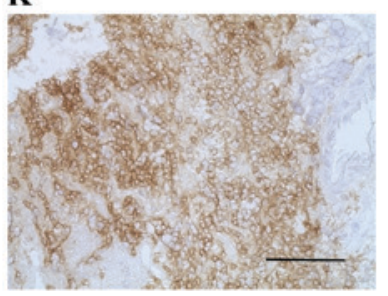

CD8

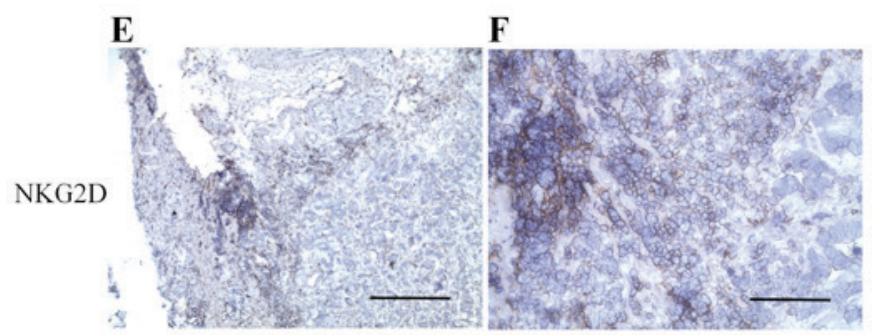

G

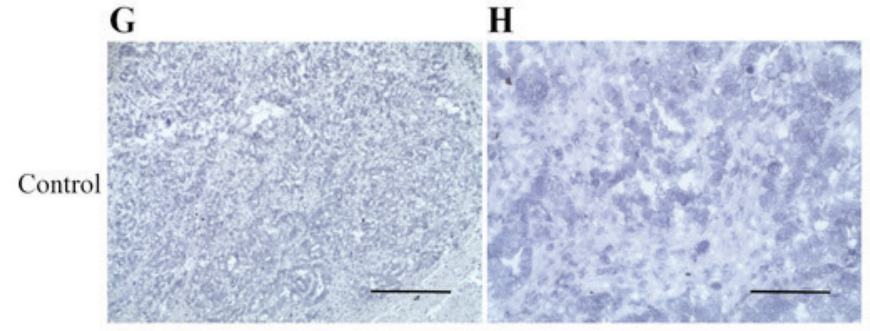

J

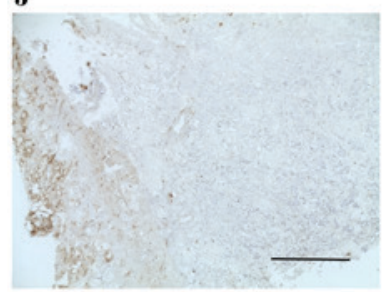

L

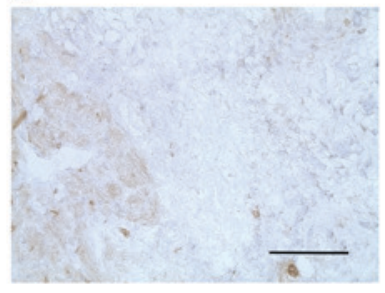

CD56

Figure 2. Representative histopathological images showing immunohistochemical staining of ULBP1, NKG2D, CD8 and CD56 in gastric cancer tissues. (A) High ULBP1 expression in gastric cancer tissues. ULBP1 expression is revealed in the cell membrane and cytoplasm (brown staining). (B) High ULBP1 expression in gastric cancer tissues. Magnification, x400; scale bar, $100 \mu \mathrm{m}$. (C) Isotype control for staining of ULBP1. Magnification, x100; scale bar, $200 \mu \mathrm{m}$. (D) Isotype control for staining of ULBP1. Magnification, x400; scale bar, $100 \mu \mathrm{m}$. (E) High NKG2D expression in tumor-infiltrating lymphocytes. Magnification, x100; scale bar, $200 \mu \mathrm{m}$. (F) High NKG2D expression in tumor-infiltrating lymphocytes. Magnification, x400; scale bar, 100 $\mu \mathrm{m}$. (G) Isotype control for staining of tumor-infiltrating lymphocytes. Magnification, x100; scale bar, $200 \mu \mathrm{m}$. (H) Isotype control for staining of tumor-infiltrating lymphocytes. Magnification, x400; scale bar, $100 \mu \mathrm{m}$. (I) Staining of CD8-positive cells in gastric cancer. Magnification, x100; scale bar, 200 $\mu \mathrm{m}$. (J) Staining of CD8-positive cells in gastric cancer tissues. Magnification, x400; scale bar, $100 \mu \mathrm{m}$. (K) Staining of CD56-positive cells. Magnification, x100; scale bar, $200 \mu \mathrm{m}$. (L) Staining of CD56-positive cells. Magnification, x400; scale bar, $100 \mu \mathrm{m}$. Immunohistochemical detection of CD8-positive or CD56-positive mononuclear cells around gastric tumors in I-L used the same sections as those in E-H. ULBP, UL16 binding protein; CD, cluster of differentiation; NKG2D, natural killer group 2 member D.

(magnification, $\mathrm{x} 100$ and $\mathrm{x} 400$, respectively) in the same sections as those used in Fig. 2E and F. Of the 98 samples, 19 were positive for NKG2D.

IHC staining of $N K G 2 D^{+}, C D 8^{+}$, and $C D 56^{+}$. Among the NKG2D-expressing tissue sections, a total of 6 samples were stained for CD8 and CD56 and were used as representative sections. Fig. 2I-L displays CD8 or CD56 expression in the same samples as those analyzed in Fig. 2E-H. In all tissues, the majority of NKG2D-expressing cells were $\mathrm{CD} 8^{+} \mathrm{T}$ cells.

Expression of ULBPI and NKG2D and clinicopathological findings. Univariate analysis indicated that NKG2D expression in mononuclear cells was associated with lymphatic invasion $(\mathrm{P}=0.0028)$ and postoperative recurrence $(\mathrm{P}=0.0048$; Table II). No significant associations were observed between ULBP1 expression and pathological findings or postoperative recurrence (Table II).

Survival curves following surgery were compared between high and low ULBP1 and NKG2D expression groups. A total of 70 patients were positive for ULBP1, whereas 28 patients were negative for ULBP1 (Table II). By contrast, a total of 19 and 79 patients were positive and negative for NKG2D, respectively (Table II). There were no significant differences in OS observed according to ULBP1 expression ( $\mathrm{P}=0.334$; Fig. $3 \mathrm{~A}$ ). However, patients with NKG2D expression were indicated 
Table II. Univariate analysis of overall survival of patients with gastric cancer following gastrectomy.

\begin{tabular}{|c|c|c|c|c|c|c|c|}
\hline \multirow[b]{2}{*}{ Parameters } & \multirow[b]{2}{*}{ Cases, $\mathrm{n}$} & \multicolumn{3}{|c|}{ ULBP1 } & \multicolumn{3}{|c|}{ NKG2D } \\
\hline & & $\begin{array}{l}\text { Positive } \\
(\mathrm{n}=70)\end{array}$ & $\begin{array}{l}\text { Negative } \\
(\mathrm{n}=28)\end{array}$ & P-value & $\begin{array}{l}\text { Positive } \\
(\mathrm{n}=19)\end{array}$ & $\begin{array}{l}\text { Negative } \\
(\mathrm{n}=79)\end{array}$ & P-value \\
\hline Depth of tumor invasion (T) & & & & 0.076 & & & 0.132 \\
\hline T2 (muscularis propria or subserosa) & 46 & 37 & 9 & & 12 & 34 & \\
\hline T3 (serosal exposure), T4 (invasion) & 52 & 33 & 19 & & 7 & 45 & \\
\hline Histological classification of gastric tumors & & & & 0.814 & & & 0.784 \\
\hline Papillary adenocarcinoma/tubular adenocarcinoma & 30 & 21 & 9 & & 5 & 26 & \\
\hline $\begin{array}{l}\text { Poorly differentiated adenocarcinoma/signet-ring } \\
\text { cell carcinoma/ mucinous adenocarcinoma }\end{array}$ & 68 & 49 & 19 & & 14 & 53 & \\
\hline Cancer stromal volume & & & & 0.647 & & & 1.000 \\
\hline Medullary type/interstitial connective tissue & 49 & 36 & 13 & & 9 & 40 & \\
\hline Scirrhous type & 48 & 29 & 14 & & 9 & 39 & \\
\hline Tumor infiltrative pattern into the surrounding tissues & & & & 0.819 & & & 1.000 \\
\hline $\mathrm{a}, \mathrm{b}$ & 38 & 28 & 10 & & 7 & 31 & \\
\hline $\mathrm{c}$ & 60 & 42 & 18 & & 12 & 48 & \\
\hline Lymphatic invasion & & & & 0.669 & & & 0.003 \\
\hline 0 & 7 & 6 & 1 & & 5 & 2 & \\
\hline $1,2,3$ & 91 & 64 & 27 & & 14 & 77 & \\
\hline Venous invasion & & & & 0.798 & & & 0.066 \\
\hline 0 & 23 & 16 & 7 & & 8 & 15 & \\
\hline $1,2,3$ & 75 & 54 & 21 & & 11 & 64 & \\
\hline Lymph node metastasis & & & & 0.461 & & & 0.776 \\
\hline 0 & 27 & 21 & 6 & & 6 & 21 & \\
\hline $1,2,3$ & 71 & 49 & 22 & & 13 & 58 & \\
\hline Stage grouping & & & & 0.262 & & & 0.311 \\
\hline I, II & 41 & 32 & 9 & & 10 & 31 & \\
\hline III, IV & 57 & 38 & 19 & & 9 & 48 & \\
\hline Stage grouping & & & & 0.111 & & & 0.549 \\
\hline I, II, III & 75 & 57 & 18 & & 16 & 59 & \\
\hline IV & 23 & 13 & 10 & & 3 & 20 & \\
\hline Postoperative recurrence & & & & 0.799 & & & 0.005 \\
\hline Presence & 33 & 25 & 8 & & 2 & 31 & \\
\hline Absence & 45 & 32 & 12 & & 15 & 30 & \\
\hline
\end{tabular}

ULBP1, UL16 binding protein 1; NKG2D, natural killer group 2 member D.

to have significantly longer OS compared with patients without NKG2D expression ( $\mathrm{P}=0.0217$; Fig. 3B). Combined expression of NKG2D and ULBP1 was also analyzed and are as follows: ULBP1-high/NKG2D-high $(\mathrm{n}=12)$, ULBP1-high/NKG2D-low ( $\mathrm{n}=58$ ), ULBP1-low/NKG2D-high $(n=7)$, and ULBP1-low/NKG2D-low $(n=21)$. With regards to OS, the findings indicated that the ULBP1-high/NKG2D-high group had the best prognosis, and the ULBP1-low/NKG2D-low group had the poorest prognosis $(\mathrm{P}=0.0096$; Fig. 3C).

Multivariate analysis. Multivariate analysis was used to determine the hazard ratios for OS conferred by ULBP1, NKG2D, sex, lymph node metastasis, lymphatic invasion and venous invasion. NKG2D expression and lymph node metastasis were identified to be independent prognostic factors for OS $(\mathrm{P}=0.0431$ and $\mathrm{P}=0.0030$, respectively; Table III).

\section{Discussion}

The present study examined the association between prognosis and expression of NKG2D and ULBP1. The results indicated that detecting a combination of ULBP1 and NKG2D expression might be an effective strategy for prediction of OS in patients with gastric cancer, and therefore this may provide important insights into the roles of these two proteins in gastric cancer. A number of solid tumors constitutively express NKG2DLs and are receptive to NKG2D-dependent immunosurveillance (6). However, soluble NKG2DLs are 
Table III. Multivariate proportional hazards analysis of overall survival patients with gastric cancer following gastrectomy.

\begin{tabular}{lccc}
\hline Variable & $\begin{array}{c}\text { Hazard } \\
\text { ratio }\end{array}$ & $95 \%$ CI & P-value \\
\hline Lymph node metastasis & 1.5670 & $1.1640-2.1100$ & 0.0030 \\
NKG2D expression & 0.3369 & $0.1173-0.9672$ & 0.0431 \\
\hline
\end{tabular}

CI, confidence interval; NKG2D, natural killer group 2 member D.
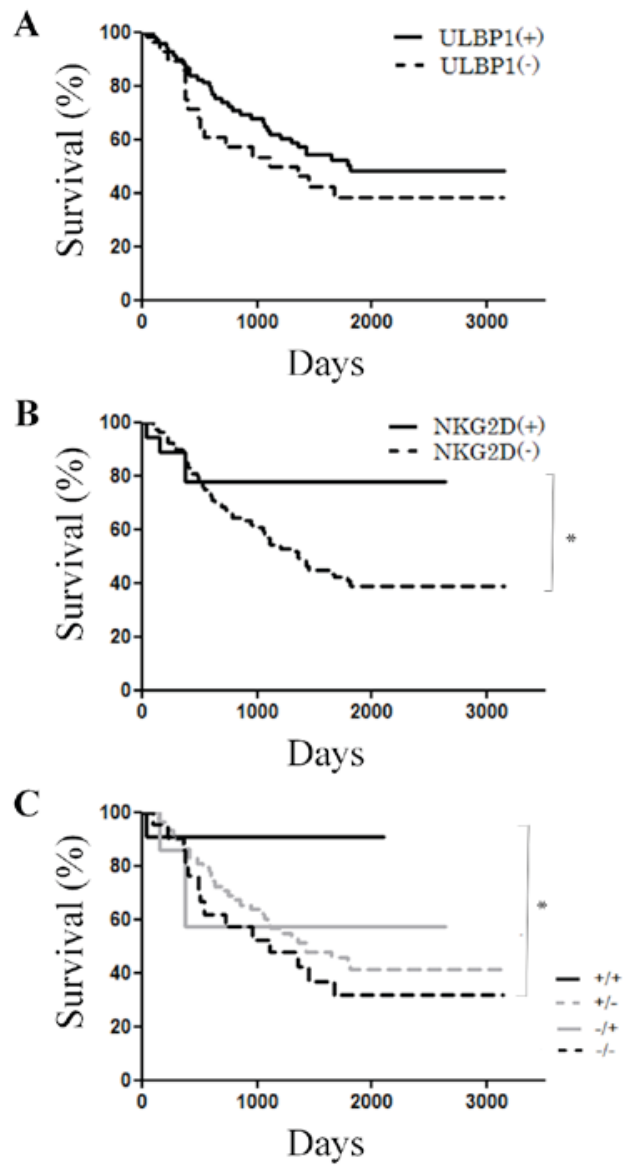

Figure 3. (A) OS in patients with high (+) or low (-) ULBP1 expression. (B) OS in patients with high (+) or low (-) NKG2D expression ( $\left.{ }^{*} \mathrm{P}=0.0217\right)$. (C) OS in patients with combinations of ULBP1 and NKG2D expression. +/+ represents patients with high expression levels of ULBP1 and NKG2D. +/-represents patients with high ULBP1 and low NKG2D expression levels. -/+ represents patients with low ULBP1 and high NKG2D expression levels. -/- represents patients with low ULBP1 and NKG2D expression levels. There is a significant difference between $+/+$ and $-/-\left({ }^{*} \mathrm{P}=0.0096\right)$. OS, overall survival; ULBP, UL16 binding protein; NKG2D, natural killer group 2 member D

released from tumors and block NKG2D activation, resulting in tumor immune escape (6). It has been demonstrated that the levels of soluble NKG2DLs in serum affect tumor progression (6). In addition, MICA shed from the cell surface may affect NKG2D/NKG2DL interactions (6). No prior studies had examined the role of the soluble form of ULBP1. In the present study, ULBP1 did not exhibit any antagonistic effects on tumor immunity through NKG2D expression.
NKG2DLs are often expressed in breast cancer tissues (13). In particular, MIC-A/B and ULBP2 expression is associated with an improved prognosis in patients with cancer (13) and immunological function of NKG2DLs have not been demonstrated (13). However, other studies have suggested that soluble MICA is involved in tumor immune escape (9). In the present study, ULBP1 expression in gastric cancer may have acted as a positive ligand by binding to NKG2D, thereby prognoses are improved when these proteins were co-expressed. Additionally, it was suggested in Osaki et al (14) that decreased NKG2D expression may be responsible for immune evasion by tumors in gastric cancer, which is consistent with the results of the present study in which patients with low NKG2D expression exhibited poorer survival.

The present study revealed that the interaction of NKG2DLs and NKG2D was involved in cancer and T-cell activation in tumors. This interaction is also thought to be important for NK activation in cancer (3). Therefore, the NKG2D/ULBP1 interaction may be involved in the association between cancer and T cells. ULBP1 and NKG2D, and the ULBP1/NKG2D interaction, may be molecular targets for immunotherapy. In patients with advanced non-small-cell lung cancer, melanoma and renal-cell cancer, the inhibition of programmed death ligand 1 (PD-L1) exhibited a robust response and blocked disease progression (15). Anti-PD-1 antibodies produced objective responses in $20-25 \%$ of patients with non-small-cell lung cancer, melanoma or renal-cell cancer (16). Therefore, one approach for blocking the ULBP1/NKG2D interaction is to use specific antibodies against ULBP1 and NKG2D, which may target T cells at sites of solid tumors, and therefore accelerates the activation of $\mathrm{T}$ cells. Additional studies are required to determine the effects of the ULBP1/NKG2D interaction on the adaptive immune system.

Therefore, the interaction between ULBP1 and NKG2D in gastric cancer may be associated with OS. In addition, the results of the present study suggested that ULBP1 and NKG2D may be applied in immunotherapy to induce acquired immunity in patients with cancer and for the prediction of prognosis following surgical resection in patients with gastric cancer. The detailed interactions between NKG2DL and NKG2D require additional investigation. The development of NKG2DL-targeted immunotherapy may have important future clinical applications.

\section{Acknowledgements}

The present study was supported in part by the National Cancer Center Research and Development Fund (grant no. 27-A-2). The authors would like to thank Ms Akiko Sano (Yamaguchi University, Ube, Japan) for technical support. The authors would also like to thank Editage (Tokyo, Japan) and Ms Manami Kobayashi (National Cancer Center, Tokyo, Japan) for assistance with English language editing.

\section{References}

1. Edge S, Byrd DR, Compton CC, Fritz AG, Greene FL and Trotti A: AJCC Cancer Staging Manual. Springer, 2010.

2. Houchins JP, Yabe T, McSherry C and Bach FH: DNA sequence analysis of NKG2, a family of related cDNA clones encoding type II integral membrane proteins on human natural killer cells. J Exp Med 173: 1017-1020, 1991. 
3. Bauer S, Groh V, Wu J, Steinle A, Phillips JH, Lanier LL and Spies T: Activation of NK cells and T cells by NKG2D, a receptor for stress-inducible MICA. Science 285: 727-729, 1999.

4. Raulet DH: Roles of the NKG2D immunoreceptor and its ligands. Nat Rev Immunol 3: 781-790, 2003.

5. González S, López-Soto A, Suarez-Alvarez B, López-Vázquez A and López-Larrea C: NKG2D ligands: Key targets of the immune response. Trends Immunol 29: 397-403, 2008.

6. Chitadze G, Bhat J, Lettau M, Janssen O and Kabelitz D Generation of soluble NKG2D ligands: Proteolytic cleavage, exosome secretion and functional implications. Scand J Immunol 78: 120-129, 2013.

7. Saito H, Osaki T and Ikeguchi M: Decreased NKG2D expression on NK cells correlates with impaired NK cell function in patients with gastric cancer. Gastric Cancer 15: 27-33, 2012.

8. Zafirova B, Wensveen FM, Gulin M and Polić B: Regulation of immune cell function and differentiation by the NKG2D receptor. Cell Mol Life Sci 68: 3519-3529, 2011.

9. Holdenrieder S, Stieber P, Peterfi A, Nagel D, Steinle A and Salih HR: Soluble MICA in malignant diseases. Int J Cancer 118: 684-687, 2006

10. Zhang C, Zhang J, Niu J, Zhou Z, Zhang J and Tian Z: Interleukin-12 improves cytotoxicity of natural killer cells via upregulated expression of NKG2D. Hum Immunol 69: 490-500, 2008.

11. Japanese Gastric Cancer Association: Japanese classification of gastric carcinoma-2nd english edition. Gastric Cancer 1: 10-24, 1998.
12. Loos M, Hedderich DM, Ottenhausen M, Giese NA, Laschinger M, Esposito I, Kleeff J and Friess H: Expression of the costimulatory molecule B7-H3 is associated with prolonged survival in human pancreatic cancer. BMC Cancer 9: 463, 2009.

13. de Kruijf EM, Sajet A, van Nes JG, Putter H, Smit VT, Eagle RA, Jafferji I, Trowsdale J, Liefers GJ, van de Velde CJ and Kuppen PJ: NKG2D ligand tumor expression and association with clinical outcome in early breast cancer patients: An observational study. BMC Cancer 12: 24, 2012.

14. Osaki T, Saito H, Yoshikawa T, Matsumoto S, Tatebe S, Tsujitani S and Ikeguchi M: Decreased NKG2D expression on $\mathrm{CD}^{+} \mathrm{T}$ cell is involved in immune evasion in patients with gastric cancer. Clin Cancer Res 13: 382-387, 2007.

15. Brahmer JR, Tykodi SS, Chow LQ, Hwu WJ, Topalian SL, Hwu P, Drake CG, Camacho LH, Kauh J, Odunsi K, et al: Safety and activity of anti-PD-L1 antibody in patients with advanced cancer. N Engl J Med 366: 2455-2465, 2012.

16. Topalian SL, Hodi FS, Brahmer JR, Gettinger SN, Smith DC, McDermott DF, Powderly JD, Carvajal RD, Sosman JA, Atkins MB, et al: Safety, activity, and immune correlates of anti-PD-1 antibody in cancer. N Engl J Med 366: 2443-2454, 2012. International (CC BY-NC-ND 4.0) License. 